\title{
Dynamic near-infrared carbon dioxide leak visualization detection during surgery using the FLIR GF343 optical imaging system
}

\author{
Mohammad Faraz Khan ${ }^{1} \cdot$ Jeffrey Dalli $^{1} \cdot \operatorname{Ronan}$ A. Cahill ${ }^{1}$ D
}

Received: 3 June 2020 / Accepted: 30 September 2020 / Published online: 9 October 2020

○) Springer Science+Business Media, LLC, part of Springer Nature 2020

There is ongoing concern regarding the aerosolization hazard of laparoscopy since the outbreak of the COVID19 pandemic [1-4]. As a global crisis without precedent in the modern surgical era, expert opinion and theoretical extrapolations dominated initial considerations and directions regarding operative care processes. As elective surgical care restarts again, it's imperative that we advance in a systemic, scientific way to fortify minimally invasive surgical practice against further waves of this or other airborne pathogens and pollutants [5]. The fundamental driver of any higher risk of intraabdominal pathogen aerosolization by laparoscopy versus laparotomy is the use and leakage of surgical gas, specifically carbon dioxide $\left(\mathrm{CO}_{2}\right)$ [6]. We set out to develop a reliable model to determine intraoperative unfiltered $\mathrm{CO}_{2}$ leak into the operating room from out of the patient to enable comprehensive understanding and address of this substantial issue. A practical and clinically deployable methodology for leak ascertainment would allow immediate feedback for surgeons intraoperatively regarding factors within their control (e.g., leaks related to skin incision or instrument usage including leaky port seals and uncapped irrigation channels) that otherwise often go unnoticed. It also would allow surgeons share best practice with evidential support and provide reassurance to centers who do not have a significant problem with $\mathrm{CO}_{2}$ leaks. Finally, it would enable rapid testing and iterative development of the many potential adjunctive mechanical solutions (e.g., improved trocar seals and valves, gas leak traps, etc.) including reproducibility in assessment and trialing.

Electronic supplementary material The online version of this article (https://doi.org/10.1007/s00464-020-08071-9) contains supplementary material, which is available to authorized users.

Ronan A. Cahill

ronan.cahill@ucd.ie

1 UCD Centre for Precision Surgery, University College Dublin and Department of Surgery, Mater Misericordiae University Hospital, 47 Eccles Street, Dublin 7, Ireland
To do this, we have examined optical gas imaging in both the experimental and clinical settings. As carbon dioxide $\left(\mathrm{CO}_{2}\right)$ is both constitutively different to room air and becomes humidified and warmed after abdominal insufflation, it can be visually exploited in a manner similar to industrial gas leak-testing either via density- or via spectral-based imaging. Visual assessment enables a "seeing is believing" approach while also enabling computational analytics and modeling of particle trajectory benchmarking against established fluid mechanical models to demonstrate droplet behavior within the gas vortex. As the operating room environment is temperature controlled and contains positive pressure ventilation, study at table-side in the realworld is crucial in any such modeling.

Among the various methods tried, near-infrared $\mathrm{CO}_{2}$ detection has been found to be a suitable method for intraprocedural case observation being both easy and reproducible in use. We have used the FLIR GF 343 (an optical spectral system manufactured by FLIR Systems Ltd. that visualizes $\mathrm{CO}_{2}$ with high sensitivity through the detection of this gas's characteristic wavelength absorption of the infrared energy being emitted from background objects) alongside a variety of interventions including both emergency and elective operations performed by both standard and robotically assisted laparoscopy (see Fig. 1 and Videos 1 \& 2) and endoscopy. Both patients and staff easily understood the concept and provided consent readily and the 'gas filming' did not disrupt any aspect of the clinical care pathway in this initial pilot of ten procedures (comprising colorectal, hepatobiliary, gyneoncological oncology, and urological surgery and upper gastrointestinal endoscopy involving 20 different consultant specialists and surgical trainees). In each case, the visual information was welcomed by the surgeons and nurses and inspired intraoperative correction of leaks where possible. It also has provoked practical, actionable suggestions regarding both protocol and technique adjustment as well as technology redesign. The clinical experience validated and expanded our preliminary experimental 


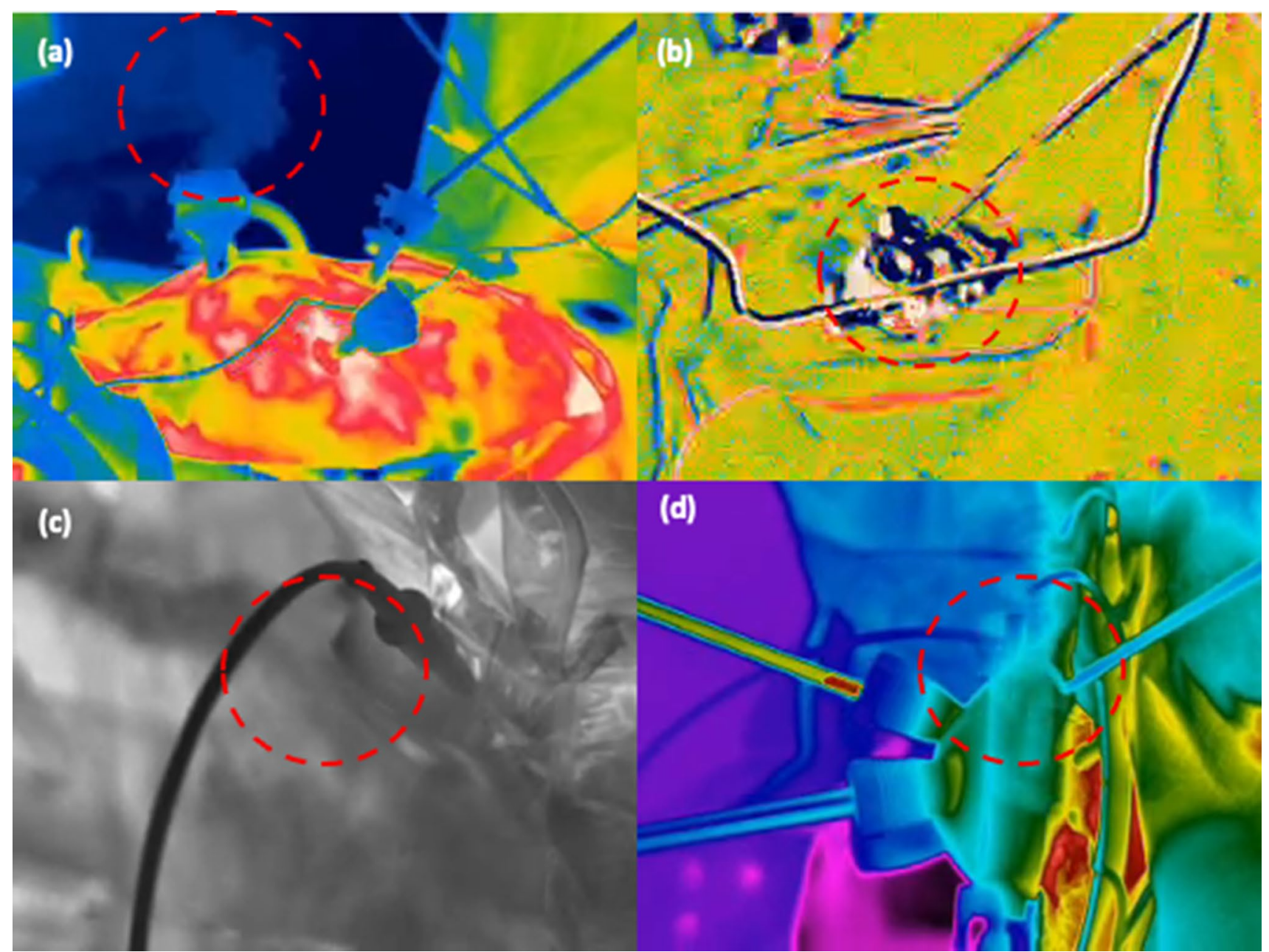

Fig. 1 Stills from associated videos showing FLIR GF343 imaging demonstrating $\mathrm{CO}_{2}$ leak (broken, red circle) from A Airseal device (Conmed), B Trocar valve on Instrument Insertion, C Robotic Scis-

framework classifying $\mathrm{CO}_{2}$ leaks into three mechanistic categories: Intentional—done by surgeon choice, for example to vent smoke or empty pneumoperitoneum at procedure end; Inadvertent-unintentional leaks caused by the surgeon, for example oversizing of trocar incisions or stretch of incisions during exertional instrument use intraoperatively; and Inbuilt-at present unavoidable by surgeon behavior, for example the gas leaks that occur through laparoscopic or robotic instrumentation due, for example, to hollow spaces in the instrument shafts [7].

Engagement with standardized methodology empowers surgeons. In this case, visual identification of gas leaks allows immediate realization and consideration of the fundamental issues. It is too a powerful teaching aid in its vivid illustration of areas of technical imperfection to help instill best practice habits at an early stage. By understanding the aerosol permeability of instrumentation, surgeons and industry partners can action design solutions for common surgical sors (Da Vinci Si), and D Gelseal Cap of Gelpoint Path (Applied Medical) (Color figure online)

instruments that can then be easily and practically tested to perfection.

Acknowledgements The authors thank Mr David Doyle of Butler Technologies, Ireland, and Mr Stephen Beynon of FLIR Systems Ltd, UK, for making the FLIR GF 343 available for this work and for providing training in its use.

\section{Compliance with ethical standards}

Disclosures Ronan A. Cahill receives speaker fees from Stryker, Consultancy Fees from Touch Surgery and Distalmotion and Research funding from Intuitive and with IBM Ireland. Mohammad Faraz Khan and Jeffrey Dalli have no conflicts of interest or financial ties to disclose. 


\section{References}

1. Francis N, Dort J, Cho E, Feldman L, Keller D, Lim R, Mikami D, Phillips E, Spaniolas K, Tsuda S, Wasco K, Arulampalam T, Sheraz M, Morales S, Pietrabissa A, Asbun H, Pryor A (2020) SAGES and EAES recommendations for minimally invasive surgery during COVID-19 pandemic. Surg Endosc. https://doi. org/10.1007/s00464-020-07565-w

2. Vigneswaran Y, Prachand VN, Posner MC, Matthews JB, Hussain M (2020) What is the appropriate use of laparoscopy over open procedures in the current COVID-19 climate? J Gastrointest Surg. https://doi.org/10.1007/s11605-020-04592-9

3. Saverio S, Di Khan M, Pata F, Ietto G, Simone B, De Zani E, Carcano G (2020) Laparoscopy at all costs? Not now during COVID19 outbreak and not for acute care surgery and emergency colorectal surgery: a practical algorithm from a hub tertiary teaching hospital in Northern Lombardy, Italy. J Trauma Acute Care Surg 88(6):715-718. https://doi.org/10.1097/TA.0000000000002727
4. Schwarz L, Tuech JJ (2020) Is the use of laparoscopy in a COVID19 epidemic free of risk? Br J Surg. https://doi.org/10.1002/ bjs. 11649

5. Mayol J, Fernández Pérez C (2020) Elective surgery after the pandemic: waves beyond the horizon. Br J Surg. https://doi. org/10.1002/bjs. 11688

6. Brat GA, Hersey S, Chhabra K, Gupta A, Scott J (2020) Protecting surgical teams during the COVID-19 outbreak: a narrative review and clinical considerations. Ann Surg. https://doi.org/10.1097/ SLA.0000000000003926

7. Cahill RA, Dalli J, Khan M, Flood M, Nolan K (2020) Solving the problems of gas leakage at laparoscopy. Br J Surg. https://doi. org/10.1002/bjs.11977

Publisher's Note Springer Nature remains neutral with regard to jurisdictional claims in published maps and institutional affiliations. 